\title{
Sur la mécanique du mouvement des matériaux solides
}

\section{On the mechanics of solid transport}

\author{
par DR. Ing. SemM YALIN
}

BUNDESANSTALT FUn WASSEHBAU. KARLSHUHE

\begin{abstract}
La presente étude tente d'aborder par voie théorique le problème du mouvement des matériaux solides si souvent étudié par voie expérimentale, et de chercher ainsi $\dot{a}$ interpréter la structure phlysique de ce phénomène. Pour cela, l'auteur met d'abord en évidence l'influence du conrant (réaction du courant) sur les grains d'un lit mobile et ensuite, compte tenu de la condition d'équilibre des forces agissant sur les éléments du lit et des résultats spéciaux tirés des essais, il dégage l'équation nécessaire à l'expression du début d'entrainement des grains. Dans la suite, il établit le système des équations différentielles caractérisant un grain mobile, lesquelles, une fois intégrées, définissent d'une part la structure cinématique des mouvements des grains et, d'autre part, la vitesse moyenne d'un grain en mouvement, en fonction de leurs arguments.

La derniere partie de l'étude est consacrée à un essai de déterminer théoriquement, $a$ partir des résultats déjà obtenus, l'expression nécessaire pour le débit solide d'un courant bi-dimensionnel. La forme du résultat obtenu correspond entièrement à celle de la formule de Meyer-Peter.
\end{abstract}

La théorie - proposée à la fin du dernier siècle par Du Buat -, du mouvement «stratifié » des matériaux solides qui serait constitué d'un mouvement de couches de fond superposées dont les vitesses décroissent linéairement en fonction de la profondeur, était jusqu'à tout récemment $\left(^{*}\right)$ la seule représentation de la structure cinématique de ce phénomène. Ce n'est que tout dernièrement que l'on a pu obtenir une image correcte de ce phénomène, et ce, au moyen d'un grand nombre d'observations et de prises ciné-

( ) Voir par exemple Rouse : Fluid Mechanies for Hydraulic Engineers, 1938.

\begin{abstract}
This work is an attempt towards a theoretical understanding of solid transport-which has already been the subject of much experimental research-and to find an interpretation for the mechanical structure of the process. With this in view, the first part of the worli concerns itself with bringing the effect of the current upon the grains in a moving river bed-i.e. the current reaction-into evidence. The author then considers the equilibrium condition for the forces acting on the bed, together with the special test results, and arrives at the equation required for expressing incipient grain entrainment. Following this, he establishes the system of differential equations for a moving grain, which, when integrated, give both the kinematic structure of the grain movements and the mean velocity of a moving grain in terms of their arguments.

The last part of the work is an attempt to find the necessary expression for the solid discharge in a two-dimensional flow by theoretical methods, starting from the results obtained so far. The form of the result obtained is seen to agree completely with that of Meyer-Peter's formula.
\end{abstract}

matographiques effectuées systématiquement en canal vitré $\left({ }^{*}\right)$.

Ces essais ont démontré que le mouvement global d'un fond (d'un ordre de grandeur prédominant dans la pratique) ne se produit que dans sa couche supérieure, où les éléments (grains) se déplacent soit en roulant (comme les gros grains du mélange de matériaux de fond aux faibles vitesses d'écoulement), soit, ce qui est généralement le cas, en franchissant chacun une certaine

(*) M. Welikanoff : Dynamique des écoulements dans les canaux, t. II (Sédiments et lit des cours d'eau), Moscou, 1955 , p. 25. 
distance par «bonds», avec des intervalles d'arrêt irréguliers; le mouvement permanent de la couche supérieure est donc formé de l'ensemble de ces mouvements individuels. Etant donné la complexité relative du phénomène, qui comprend simultanément le mouvement et l'influence réciproque de deux phases physiques différentes, comportant de ce fait de très nombreux facteurs qui doivent caractériser ce phénomène, la tendance à l'étudier expérimentalement s'est développée. Il en résulte que l'on dispose actuellement de données expérimentales surabondantes, mais qu'il n'existe encore aucune théorie physique nécessaire qui pourrait servir utilement de base scientifique pour le traitement du phénomène du mouvement des matériaux solides. I.es formules empiriques établies jusqu'à présent pour le transport des matériaux solides, et qui ont pour objet d'exprimer la relation mathématique entre les arguments d'un phénomène physique donné, présentent le plus souvent des structures totalement différentes $\left({ }^{\star * *}\right)$, ce qui montre clairement de combien de manières différentes on peut interpréter une expérience, tant que celle-ci n'a pas été effectuée sur la base d'une théorie physique.

C'est pour cette raison que l'on a été amené à aborder dans la présente étude cet aspect théorique encore si peu traité du phénomène. Nous avons essayé d'interpréter la structure mécanique du phénomène et de trouver l'expression recherchée pour le débit solide. Il est intéressant de noter qu'à la fin de cette étude le résultat développé théoriquement se trouve en concordance complète avec la formule de Meyer-Peter.

\section{1. - REACTION DU COURANT SUR LES ELEMENTS DU FOND}

Considérons l'écoulement bi-dimensionnel, permanent, ét stationnaire d'un fluide réel au voisinage d'un fond plan et rugueux. La structure mécanique d'un mouvement défini de telle sorte est déterminée par les paramètres indépendants (1) :

$$
. k_{s} ; \rho ; \mu ; u_{*}
$$

Selon les principes de base du calcul dimensionnel généralisé (théorème des $\pi$ ), toutes les grandeurs mécaniques sans dimensions intervenant seront les fonctions de $(4-3=1)$ d'une combinaison sans dimensions composée des quatre paramètres ci-dessus, c'est-à-dire de la seule combinaison possible :

$$
\mathfrak{R}_{*}=\frac{u_{*} k_{s}}{\nu} \text { avec } \quad \nu=\mu / \rho
$$

Considérons maintenant un grain reposant sur le fond, soit $a$ ou $b$ de la figure 1 , placé directement dans l'écoulement et donc soumis à une force $\vec{F}$ (《réaction du courant») par le milieu liquide. Désignons par $F$ la grandeur de ce vec-

(**) Voil par exemple Ies formules de Du Boys, Schocklitsch, Nakajama, Tschang, Mac Dougall, Gilbert, Meyer-Peter, Donath, Poljakoff, Lewi, Gontscharoff, etc.

(1) On admet (du moins en principe) que la rugosité du fond $k$ peut toujours être remplacée par une rugosité de sable $k_{s}$ de. Nikuradse équivalente (voir pour plus de détails, par exemple, H. Schlichring: GrenzschichtTheorie, G. Bravn, KarIsruhe, 1951 (XX, g), p. 385. teur et son angle d'inclinaison par 0 . Nous n'exprimerons la grandeur géométrique du grain que par une dimension linéaire, c'est-à-dire par son diamètre $d\left(d=\alpha k_{s}\right)\left({ }^{2}\right)$.

D’après ce qui précède, toutes les grandeurs sans dimensions intervenant pour le grain, et done

$$
\lambda=\mathrm{F} / \boldsymbol{u}_{*}^{2} \rho\left(\alpha \boldsymbol{k}_{s}\right)=\text { et } 0
$$

seront les fonctions de $\mathcal{R}_{*}$ qui lui sont propres, à savoir :

$$
\left.\begin{array}{l}
\lambda=\bar{\lambda} \alpha_{*} \\
\theta=\bar{\theta} \alpha_{*}
\end{array}\right\}
$$

d'où :

$$
\mathrm{F}=\left\lceil\lambda \mathfrak{A}_{*} \alpha^{2}\right\rceil o k_{s}{ }^{2} \cdot u_{*}{ }^{2}
$$

Il est évident que la forme des relations fonctionnelles :

$$
\bar{\lambda} \mathscr{R}_{*} \quad \text { et } \quad \theta \mathrm{R}_{*}
$$

dépend de la forme géométrique des conditions aux limites de l'écoulement considéré autour du grain, e'est-à-dire de la grandeur relative, du calage en altitude, de la forme, etc... du grain même et des grains dans son voisinage, qui peuvent influencer plus ou moins nettement l'écoulement autour de lui.

(2) Pour plus de détails sur la forme sphérique des éléments de la couche mobile, voir par exemple M. WEL1KANOFF (renvoi $\left(^{*}\right.$ ) ci-dessus), t. II, p. 9. 


\section{2. - DEBUT D'ENTRAINEMENT DES MATERIAUX SOLIDES}

Les forces qui agissent sur un grain de la couche supérieure peu avant sa mise en mouvement sont les suivantes : poids du grain (pesé sous l'eau) $\vec{G}$, la réaction du courant sur le grain $\vec{F}$ et la force d'appui $\vec{R}$. Soit $\varphi$ l'angle de frottement entre grains et $\psi$ l'angle d'inclinaison des tangentes au point d'appui T-T; la condition pour
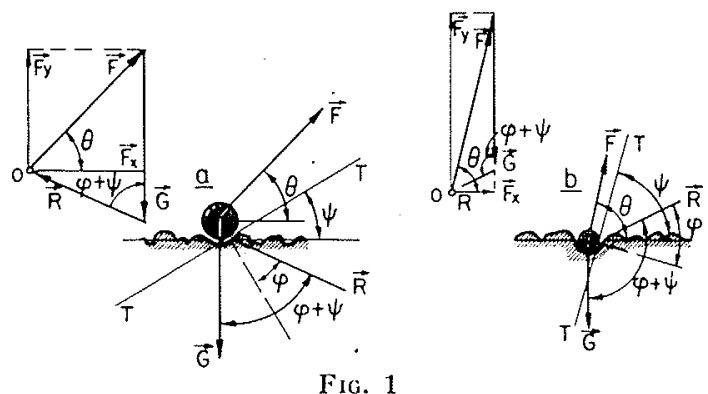

FIG. 1

la mise en mouvement du grain, suivant le triangle des forces représenté sur la figure 1, est alors :

$$
\mathbf{F}_{v} \geqq\left(\mathbf{G}-\mathbf{F}_{y}\right) \operatorname{tg}(\varphi+\varphi)
$$

Nous basant sur le sens mécanique de cette formule (3), nous appellerons le facteur :

$$
f=\operatorname{tg}(\varphi+\psi)
$$

" coefficient de frottement apparent », et la somme des angles :

$$
\varphi+\psi
$$

« angle apparent de frottement» du grain considéré.

Compte tenu de la relation trigonométrique de $\mathrm{F}_{x}, \mathrm{~F}_{y}$ et $\mathrm{F}^{x}$ par rapport à l'angle $\theta$, nous pouvons exprimer la condition (3) sous les formes suivantes :

(3) En admettant l'hypothèse plausible $k_{s} \approx d_{m}$ et en éliminant de la formule représentant le début d'entrấnement (par exemple la formule Meyer-Peter) la tension de cisaillement et compte tenu de la condition

$$
\begin{aligned}
\mathcal{G}_{*} \supseteq 70 \text { avec } g & =9,81 \mathrm{~m} / \mathrm{s}^{2}, \gamma_{s} / \gamma=1,80 \\
\text { et } \nu & =1,3.10^{-6},
\end{aligned}
$$

on a $d_{m} \geqslant 0,215 \mathrm{~cm}$. Cela veut dire que, dans la grande majorité des cas se présentant dans la nature, on ne saurait prévoir le début du mouvement qu'après « écoulement rugueux parfaitement établi $», \lambda$ cessant de ce fait de dépendre de $\mathcal{R}_{*}$ et devenant constant, ceci pour la plupart des éléments composant le lit.

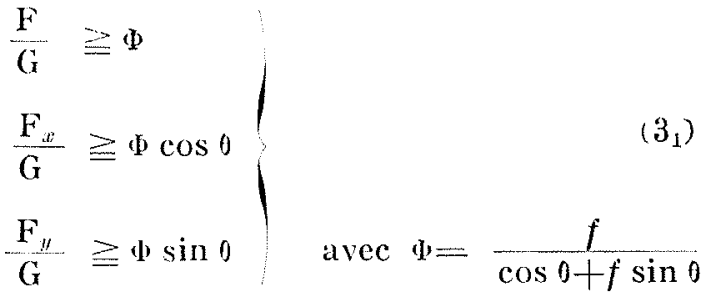

Le poids du grain sous l'eau peut être exprimé jar :

$$
\mathrm{G}=\alpha_{f} \gamma_{s} d^{3}=\left(\alpha_{j} \alpha^{3}\right) \gamma_{s} k_{s}{ }^{3}
$$

$\gamma_{s}$ étant le poiōs unitaire pesé sous l'eau (admis comme égal pour tous les grains) et $\alpha_{f}$ le coefficient de forme (pour la sphère, $\alpha_{f}=\pi / 6$ ).

Si nous substituons à $F$ et $G$ dans la première formule $\left(3_{1}\right)$ leurs valeurs $\left(2_{1}\right)$ et $(4)$, nous obtenons :

$$
\frac{\gamma}{\gamma_{s}} \cdot \frac{u_{*}^{2}}{g k_{s}} \geqq \frac{\alpha_{f} \alpha}{\bar{\lambda}\left(\mathcal{R}_{*}\right)} \Phi=W^{(3)}
$$

dont le premier membre ne dépend plus des propriétés individuelles du grain considéré. En désignant (par analogie avec $\alpha_{*}=u_{*} k_{s} / \nu$ la combinaison sans dimension $l_{*}^{2} / g k_{s}$ par $\mathscr{F}^{*} *$, on arrive à la conclusion que, pour que le grain puisse se mettre en mouvement, il faut que le nombre de Froude $\mathscr{H}_{*}$ soit suffisamment grand pour que le premier membre de la relation ci-dessus atteigne l'expression sans dimensions W propre au grain considéré, ce $W$ étant fonction du coefficient de forme, des dimensions relatives, de la « géométrie » de position du grain en liaison avec $\mathfrak{A}_{*}$, et de l'angle de frottement entre les grains, c'est-à-dire des valeurs :

$$
\alpha, \alpha, \psi, \lambda, \theta \text {, et } \varphi \text {. }
$$

Si l'on tient compte de la tendance de la relation $\lambda\left(\mathcal{R}_{*}\right)$ et des écarts insignifiants par rap-

(4) Au sujet de l'écoulement symétrique autour d'une sphère, voir H. Schlichting, ouvrage cité, renvoi 1$), 1, e$. - L. SEDOFF : Les méthodes de similitude et dimensions en mécanique, Moscou, 1954, II, 4, pp. 49-53.

Au sujet de l'écoulement non symétrique, voir M. Denentiev : Interference of two solid bodies in a stream of fluid, Transactions of the Scientific Research Institute of Hydrautechnics (V; XV), Leningrad, 1935. 
port à l'unité de la combinaison triangulaire I lorsque $\psi$ varie entre 0 et $\pi / 2$ (5) $^{(5)}$ trouve que les valeurs $W$ varient généralement en fonction de $u_{*}$ de la facon représentée par la figure 2 et que l' « état critique» d'un grain se présentera à la valeur $u_{*}=u_{* k}$. correspondant au point d'intersection de la parabole $\psi_{/} \psi_{s} \mathscr{F}_{*}$ et la courbe de $W$.

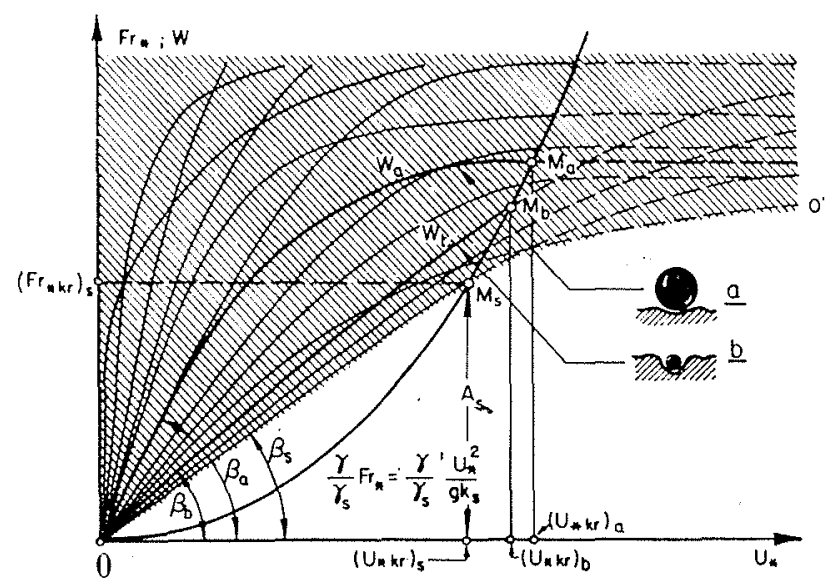

Fic. 2

Avant d'étendre ce que nous avons dit au sujet de l' « état critique » d'un grain particulier, à l'ensemble des grains de la couche supérieure, et de passer à l' "état critique d'une couche de fond $»$, nous considérerons les résultats expérimentaux susceptibles de nous assister dans cette tâche.

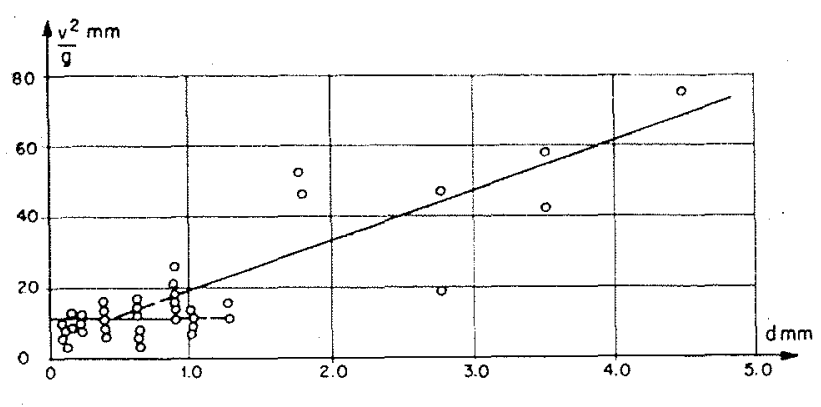

F1G. 3

La figure 3 représente les résultats d'essais ayant pour but de déterminer la relation entre la granulométrie $d$ d'un fond ( $d \approx$ constant) et les valeurs $(v)$ de la vitesse moyenne dans le canal d'essai correspondant à la mise en

(5) Pour plus de détails sur la combinaison triangulaire, voir S. YaLIN : Die theoretische Analyse der Mechanik der Geschiebebewegung, Mitteilungsblatt der Bundesanstalt für Wasserbau, Nr. 8, Karlsruhe, 1957. mouvement du fond (i). Il est intéressant de noter que la courbe expérimentale ne tend pas du tout à recouper l'origine des coordonnées. Par contre, les essais en canal vitré au laboratoire d'hydraulique fluviale Theodor Rehbock, de l'Ecole Polytechnique de Karlsruhe, ont été effectués avec un matériau de fond constitué de trois mélanges fondamentalement différents, composés des fractions :

$$
d_{\mathrm{min}} \rightarrow 0 \leqq d_{\max }=8 \mathrm{~mm}
$$

les courbes granulométriques de ces matériaux $a, b$ et $c$ sont représentées sur la figure 4 .

On a constaté que, pour une augmentation pratiquement uniforme du débit d'eau (à partir de $Q=0$ ) dans le canal d'essai, on ne pouvait observer des quantités appréciables de matériau de fond (échantillon) dans la boîte collectrice qu'à partir d'un certain régime d'écoulement, que l'on peut appeler le « régime d'apparition d'entraînement $\gg$. L'analyse au tamis de ces premiers échantillons a donné les courhes granulométriques $a_{1}, b_{1}, c_{1}$ de la figure 4 .

Nous avons déduit de ces données expérimentales que l' «état critique » déterminant le début de mouvement d'un grain quelconque (et même du plus petit) ne peut se produire que pour une valeur finie bien définie de $u_{*}(d \rightarrow 0$ ne conditionne donc pas $u_{* i r} \rightarrow 0$ ). Nous concluons en outre que, même s'il se produit un mouvement appréciable des éléments de fond (ce qui n'est pas forcément le cas), les grains participant au mouvement de masse (débit solide) représentent en général toutes les grandeurs de $d$, avec prépondérance toutefois des fractions de faible $《 d 》$ (voir les courbes $a, a_{1} ; b, b_{1} ; c, c_{1}$ ).

Nous chercherons maintenant à poursuivre notre interprétation de la structure mécanique du phénomène à la lumière de ces résultats expérimentaux. Considérons une zone suffisamment étendue, par rapport à la granulométrie des matériaux, de la surface d'un fond (vue en plan) et supposons que la couche supérieure de cette zone soit constituée de $n$ grains. Etant donné que, suivant les données expérimentales, la valeur limite $u_{* k r} \rightarrow 0$ ne peut exister en pratique pour aucun grain, nous concluons que toutes les courbes de $W$ doivent se trouver dans une zone déterminée (zone hachurée sur la figure 2), dont la limite inférieure $\overparen{00^{\prime}}$ a une inclinaison initiale de $\beta_{s}>0$.

La pente de la parabole $\mathscr{F}_{*}($ fig. 2 ) se raidit en fonction de valeurs croissantes de $n_{*}$ et pénètre

(6) M. Werikanofr, ouviage cité renvoi $\left({ }^{\star \star}\right)$ ci-dessus, t. II, p. 23. 
dans la zone des courbes de W à l'endroit où elle recoupe ces courbes aux points de $\mathscr{F}_{*}=\mathrm{W}$, qui définissent l' « état critique » (début de mouvement) des grains correspondants.

Le mouvement de masse des éléments de fond (début d'entraînement des matériaux solides) se déclenche donc à la valeur de $u_{*}=\left(u_{* k r}\right)_{s}$ correspondant à la pénétration de la parabole $\left(\gamma / \gamma_{s}\right) \mathscr{Y}_{*}$ dans la zone des courbes de W (c'est-à-dire le point d'intersection des courbes $\mathscr{F}_{*}$ et ${\overparen{00^{\prime}}}^{(7)}$. coefficient pour une forme bien déterminée des grains (sphérique) et uniquement fonction de la granulométrie du matériau de fond (c'est-à-dire de l'allure géométrique de la courbe granulométrique).

Dans le cas d'un écoulement bidimensionnel à Ja surface libre, on a :

$$
\tau_{0}=\gamma h i \text {, soit } \tau_{0}{ }_{k r}=\gamma h_{k}, i
$$

et $k_{s}$ pourra évidemment toujours ètre repré-

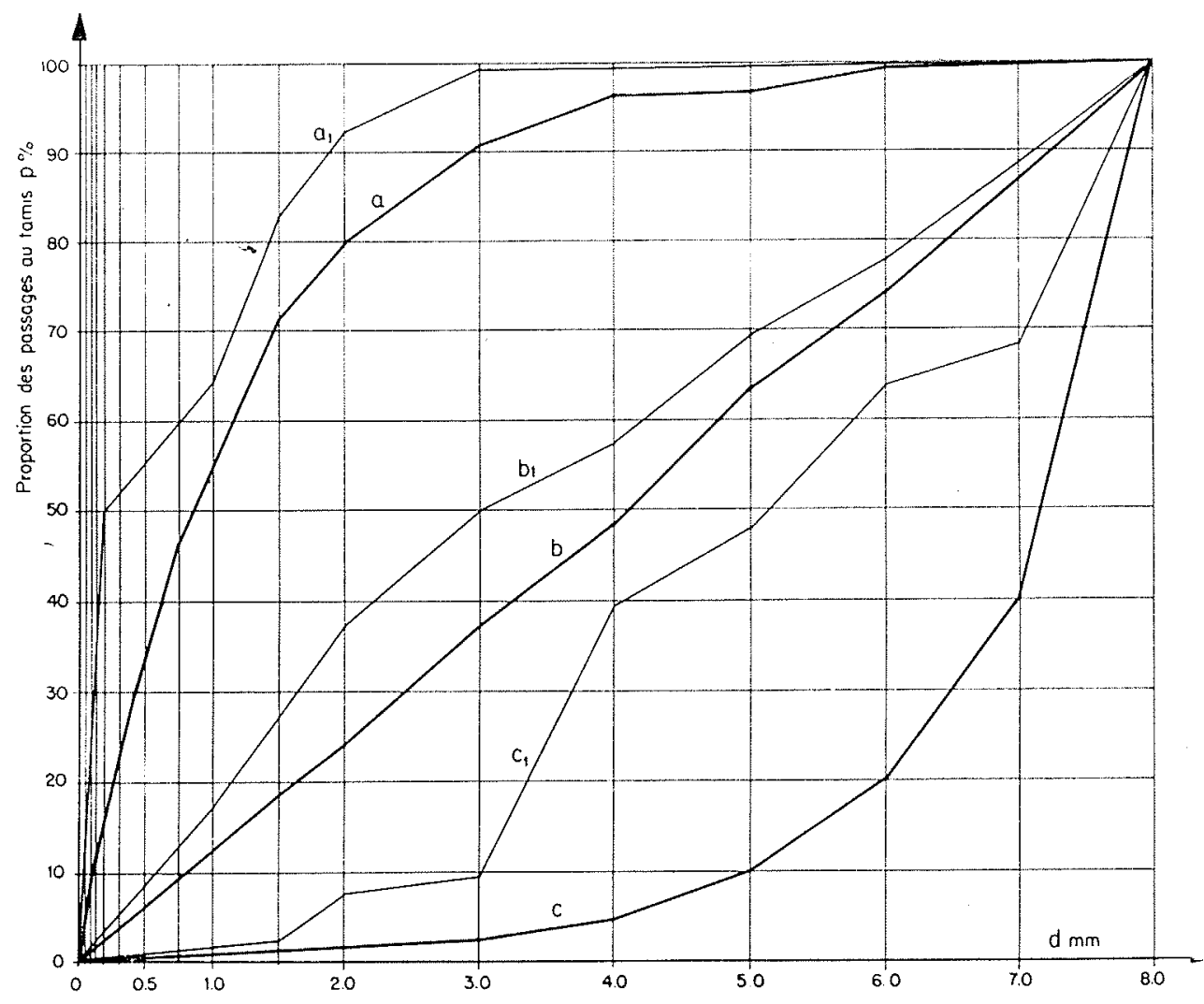

FIG. 4

Il découle de l’égalité des ordonnées en ce point :

$$
\left(\gamma / \gamma_{s}\right)\left(\mathscr{F}_{* k r}\right)_{s}=\left(\gamma / \gamma_{s}\right)\left(u_{* k r}\right)_{8}^{2} / g k_{s}=\mathrm{A}_{s}
$$

ou bien :

$$
\tau_{0 k r}=\mathrm{A}_{s} \gamma_{s} k_{s}
$$

$\tau_{0 k r}$ désignant la tension de cisaillement du fond (paroi) $\tau_{0}=u_{*}{ }^{2}$, , correspondant au début d'entraînement des matériaux solides, et $\mathrm{A}_{s}$ étant un

(7) L'indice s accompagne les valeurs qui se rapportent a l'ensemble du fond. L'indice $k r$ désigne le début du mouvement, encore ici (c'est-à-dire celui du fond). senté par le diamètre moyen des grains sous la forme $k_{s}=\alpha_{m} d_{m}$ où $\alpha_{m}$ est un nombre déterminé pour une allure donnée de la courbe granulométrique. De cette façon, on obtient de ces relations l'expression pour le début d'entrainement du matériau (dans la forme posée par MeyerPeter) :

ou bien :

$$
\left.\begin{array}{c}
\tau_{0 k r}=\mathrm{A} \gamma_{s} d_{m} \\
\gamma_{\gamma} h_{k r} i-\mathrm{A}_{\gamma_{s}} d_{m}=0
\end{array}\right\}
$$

$A=x_{m} A_{s}$ ne dépend done également que de la granulométrie du matériau de fond. 


\section{3. - MOUVEMENT DU MATERIAU SOLIDE}

Considérons maintenant les états critiques des grains $a$ et $b$ de la figure 1 , dont les angles de frottement apparents sont situés dans les zones :

$$
\begin{array}{ll}
\varphi+\psi<\pi / 2 & \text { (grain } a) \\
\varphi+\psi>\pi / 2 & (\text { grain } b)
\end{array}
$$

Lors du début du mouvement du grain $a$, la résultante $\vec{F}+\vec{G}$ est dirigée vers le bas (cas classique d'un «gros grain), c'est-à-dire que le mouvement du grain commence lorsque : $\mathrm{F}_{y}<\mathrm{G}$, de sorte que le grain se déplacera en «roulant» sans quitter la surface du fond $\left(^{8}\right)$.

Par contre, dans le cas du grain $b$, la résullante $\vec{F}+\vec{G}$ est dirigée vers le haut, de sorte que $\overrightarrow{\mathrm{F}}_{y}>\overrightarrow{\mathrm{G}}$, d'où il s'ensuit que le grain se trouve repoussé dé la surface du fond, se déplaçant en «volant» (pendant un certain temps) sans la toucher.

En principe, ces deux genres de mouvement ne se distinguent pas entre eux; autrement dit, ils ont tous deux la même structure dynamique.

En fait, le « roulement»d'un grain quelconque influence les autres corps stationnaires. Ceci signifie que la hauteur des aspérités (la rugosité) du fond est du mème ordre de grandeur que les dimensions du grain roulant. De plus, dans la nature, la répartition de ces aspérités sur la surface du fond n'est pas rigoureusement uniforme.

Par conséquent, tout «obstacle»sur le chemin du grain «roulant» provoquera une diminution soudaine de sa vitesse $\vec{c}$, c'est-à-dire une augmentation soudaine de la vitesse d'écoulement relative $\left(u-c_{x}\right)$ par rapport au grain, et, par conséquent, de la force (poussée) d'entrainement $\vec{F}$, qui croît avec cette vitesse. Il est donc évident que tout mouvement ayant l'allure d'un « roulement » s'accompagne d'augmentations (oscillations) plus ou moins impulsives du vecteur $\vec{F}$. De ce fait, le grain sera en réalité mis en

(8) L'effet de « roulement » se produit parce que (pour des raisons bien connues) te point d'application de la réaction au courant $\vec{F}$ se trouve au-dessus du centre de figure du grain, d'où s'engendre un couple de moment $\mathrm{M}=\mathrm{F} . e$, le symbole $e$ désignant l'excentration de la force $\vec{F}$. «roulement bondissant» (cf. saltation), d'où il ne saurait guère être question d'un contact permanent (continu) avec la surface de la couche de fond.

Nous passerons maintenant à l'interprétation de l'aspect mécanique de ce mouvement saltatoire des grains, que l'on peut considérer en tant que base cinétique pour le mouvement des éléments du fond, d'après ce qui précède.

A cette fin, nous commencerons par chercher à établir la relation pour la réaction du courant sur un grain en mouvement au voisinage du fond.

\section{a) Réaction du courant sur un corps (grain) en mouvement au voisinage du fond.}

Désignons la valeur instantanée du vecteur de vitesse par $\vec{c}$, et mettons $c_{x}, c_{y}$, pour ses composantes sur les axes correspondants; nous avons aiors, à l'instant considéré, la vitesse relative du courant $v$ par rapport au grain $\left({ }^{9}\right)$ :

$$
v=u-c_{x}=u_{*} \vec{u}-c
$$

ii étant en général une fonction sans dimensions de $y / k_{s}$ et de $\mathcal{R}_{*}$ (loi des parois). Partant des principes de la théorie du calcul dimensionnel, nous exprimons la valeur de la réaction du courant de la manière suivante :

$$
\mathrm{F}=\left(\lambda \alpha^{2}\right){ }_{i} k_{s}^{2}\left(u-c_{x}\right)^{2}
$$

Contrairement au cas d'un grain reposant sur la surface du fond, la géométrie instantanée des conditions aux limites pour l'écoulement autour d'un grain sphérique est entièrement caractérisée par les trois grandeurs linéaires $y, d$ et $k_{s}$.

Il faut toutefois tenir compte du fait que lorsqu'un grain «vole» sur une longueur $X$ sufsamment grande par rapport à $k_{s}$, les déformations locales des lignes d'écoulement n'ont généralement aucune influence sur la trajectoire du grain considéré, ceci étant dû à la rugosité au voisinage immédiat du fond.

(9) On pourra choisir pour v la vitesse incidente correspondant à un point typique quelconque du grain, par exemple $v_{u}, v_{m}$ ou $v_{0}$, les indices se rapportant au point inférieur, au point milieu et au point supérieur. Toutefois, puisque nous aurons à traiter dans ce qui suit le mouvement du grain comme mouvement d'un point matériel, on prendra $y_{0} \approx y_{u}=y$ et $u_{0} \approx u_{u}=u$. 
On comprend alors que l'influence de la rugosité ne se manifestera que sur la répartition des vitesses (on a déjà entièrement tenu compte de ce fait plus hant au moyen de la loi des parois) et que, par conséquent, les deux seules grandeurs linéaires $y$ et $d$ suffisent pour définir la géométrie du système considéré.

Il s'ensuit que toutes les grandeurs sans dimensions liées au mouvement considéré seront des fonctions de $\mathcal{R}$ et de $y / d{ }^{(10)}$ :

$$
\begin{aligned}
& \lambda=\bar{\lambda}(\mathcal{R} ; y / d) \\
& \theta=\bar{\theta}(\mathcal{R} ; y / d)
\end{aligned}
$$

Si nous tenons compte des relations :

$$
\left.\mathfrak{R}=x \mathcal{R}_{*}\left[\imath-c / \boldsymbol{u}_{*}\right)\right] \text { et } x(y / d)=y / k_{s}
$$

nous avons :

$$
\left.\begin{array}{l}
\lambda=\lambda\left(\mathcal{R}_{*} ; y / k_{s} ; d / k_{s} ; c / u_{*}\right) \\
0=0\left(\mathcal{R}_{*} ; y / k_{s} ; d / k_{s} ; c / u_{*}\right)
\end{array}\right\}
$$

\section{b) Le (vol " (saltation) d'un grain.}

Suivant le principe de base de la dynamique, les forces extérieures $\vec{F}, \vec{G}$ et la force d'inertie $\overrightarrow{\mathrm{Q}}=-(G / g)(\overrightarrow{d c} / d t)$ sont reliées entre elles à lout instant pendant le « vol » du grain par la relation :

$$
\overrightarrow{\mathrm{F}}+\overrightarrow{\mathrm{G}}+\overrightarrow{\mathrm{Q}}=0
$$

Les projections de cette expression vectorielle (11) sur les axes $x$ et $y$ deviennent, après une simple transformation algébrique :

$$
-\mathrm{Q}_{x}=\mathrm{F}_{x} ; \quad-\mathrm{Q}_{y}=\mathrm{F}_{y}-\mathrm{G}
$$

En introduisant les valeurs correspondantes et en appliquant une simple transformation algébrique, nous obtenons le système d'équations différentielles suivant pour le mouvement d'un grain « volant» (en saltation):

$\left.(1 / g)\left(d c_{x} / d t\right)=\cos \theta\left(\lambda / \alpha_{f}\right)\left(\gamma / \gamma_{s}\right) \mathscr{H} \cdot\left[1--\left(c_{x} / u\right)\right]^{2}\right)$

$(1 / g)\left(d c_{y} / d t\right)=\left[\operatorname{tg} 0(1 / g)\left(d c_{x} / d t\right)\right]-1$

ou :

$\begin{aligned}(1 / g)\left(d^{2} x / d t^{2}\right)=\cos \theta\left(\lambda / \alpha_{f}\right)\left(\gamma / \gamma_{s}\right) \mathscr{H} & \\ & {\left.[1-1(1 / u)(d x / d t)]^{2}\right) }\end{aligned}$

$(1 / g)\left(d^{2} y / d t^{2}\right)=\left[\operatorname{tg} \theta(1 / g)\left(d^{2} x / d t^{2}\right)\right]-1$

(10) Au sujet des valeurs expérimentales de $\lambda$ et de (et de $\lambda_{r}, \lambda_{y}$ ) fonctions de $y / d$ (pour des nombres de Reynolds élevés), voir M. Dementiev, renvoi (4).

$$
\text { (avec } \left.\mathscr{F}=u^{2} / g d=\left(\boldsymbol{u}^{*} / x\right) \mathscr{J}_{*}\right)
$$

Nous chercherons maintenant à décrire la structure mécanique d'une phase de « vol »sans avoir recours à un procédé de calcul quelconque. Immédiatement après le début du mouvement $(t=0)$ du grain dans la direction $(\vec{F}+\vec{G})$, l'accélération «par à coups »du grain provoque une diminution rapide de la grandeur du vecteur $\vec{F}$ proportionnelle à $\left(u-c_{x}\right)^{2}$, ce qui fait pivoter la résultante $\overrightarrow{(\mathbf{F}}+\overrightarrow{\mathbf{G})}$ vers le has dans le sens désigné sur la figure 5 par (-). Cette rotation de la résultante se trouve en outre accélérée par la

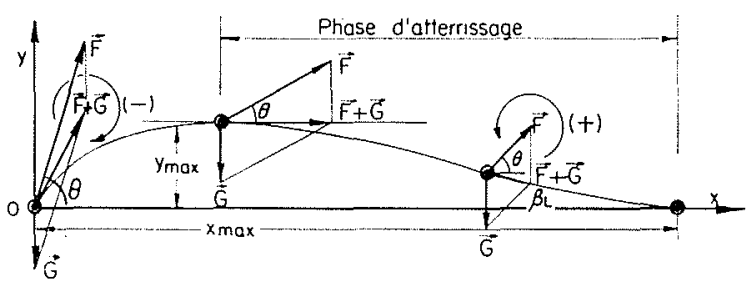

FI(4. 5)

décroissance de l'angle 0 (pour des valeurs croissantes du calage en altitude $y / d$ ). Lorsque, de cette facon, le grain atteint la position la plus élevée $(y / d)_{\max }$, l'accroissement ultérieur de $c_{x}$ (et donc la diminution de F) provoque la "phase d'atterrissage » du grain. En même temps, la réduction du calage en altitude $! / d$ provoque la croissance de l'angle $\theta$, et le vecteur $\vec{F}$, qui didiminue constamment, pivote dans le sens positif (fig. 5), ce qui fait que la courbure de la trajectoire (primitivement supposée positive) diminue à mesure qu'elle s'approche du fond, ses valeurs devenant de plus en plus petites ou même négatives dans certains cas (réduction de l'angle «d'atterrissage 》 $\beta_{\mathrm{L}}$ ). Le système d'équations différentielles (12) tel qu'il est donné plus haut (et où $\lambda, \theta$ et $u$ sont eux-mèmes des fonctions de $\mathfrak{R}=v d / v$, donc de $d x / d t$ et de $y / d$ ) ne peut être intégré par les moyens actuels de l'analyse mathématique, du moins pratiquement, sinon en principe (11). Par contre, il ne faut pas oublier que, dans les conditions régnant dans la pratique, les trajectoires des grains « en saltation $\gg$ sont si aplaties que l'on peut négliger les variations des grandeurs $\lambda, \theta$ et $u$ dues à celles des calages en altitude $y / d$, et on peut considérer celles-ci comme grandeurs indépendantes de $y$.

De plus, en supposant que la loi quadratique pour la valeur de $F$ est valable, il s'ensuit que

(11) Il n'y a même pas besoin de rappeler, à l'appui, qu'on n'a pas encore trouvé d'expression analytique capable d'exprimer les relations fonctionnelles entre $\lambda$. et $\mathfrak{\vartheta}$ et leurs arguments. 
l'influence du nombre de Reynolds, et, par suite, de $d x / d t$, sur les grandeurs $\lambda, 0$ et $u$, est éliminée sur toute la trajectoire du grain. (Les solutions que l'on obtient de cette manière ne peuvent, bien entendu, pas entièrement caractériser ceux des phénomènes de mouvement des grains qui ne remplissent pas les conditions preserites. Toutefois, ceci n'est valable dans la nature que pour les grains de très faibles dimensions, pour lesquels on ne peut admettre ni que la loi quadratique de résistance soit valable, ni que leur trajectoire soit suffisamment plate).

Nous passerons maintenant à l'intégration du système d'équations (12), en admettant que $\lambda, 0$ et $u$ soient des constantes. Posons pour abréger :

$$
\left.\cos 0\left(\lambda / \alpha_{f}\right)\right)\left(\gamma / \gamma_{s}\right) \mathscr{g}=a
$$

et introduisons les coordonnées sans dimensions :

$$
\xi=x /\left(u^{2} / g\right) ; \quad \eta=y /\left(u^{2} / g\right) ; \quad \tau=t /(u / g)
$$

avec les désignations:

$$
c_{\xi}=d \xi / d_{\tau}=c_{x} / u \quad c_{\eta}=d_{\eta} / d_{\tau}=c_{y} / u
$$

Nous obtenons :

$$
\left.\begin{array}{c}
d c_{\xi} / d \tau=a\left(1-c_{\xi}\right)^{2} \\
d c_{\eta} / d \tau=\operatorname{tg} 0\left(d c_{\xi} / d_{\tau}\right)-1
\end{array}\right\}
$$

ou bien :

$$
\left.\begin{array}{l}
d^{2 \xi} / d \tau^{2}=a[1-(d \xi / d \tau)]^{2} \\
d^{2} \eta / d \tau^{2}=\operatorname{tg} 0\left(d^{2} \xi / d \tau^{2}\right)-1
\end{array}\right\}
$$

La première de ces deux équations (13) peul être intégrée immédiatement:

$$
\int_{0}^{\tau} d \tau=(1 / a) \int_{0}^{c_{\xi}} d c_{\xi} /\left(1-c_{\xi}\right)^{2}
$$

d'où nous tirons :

$$
c_{\xi}=a \tau /(1+a \tau)
$$

Une nouvelle intégration de $c_{\xi}$ :

$$
\int_{0}^{\xi} d \xi=\int_{0}^{\tau}[a \tau /(1+a \tau)] \cdot d \tau
$$

nous donne :

$$
\xi / \tau=1-[(1 / a \tau) \ln (1+a \tau)]
$$

La deuxième des équations (13) s’intègre de la facon suivante :

$$
\int_{0}^{c} \eta d c_{\eta}=\operatorname{tg} \theta \int_{0}^{c} \xi d c_{\xi}-\int_{0}^{\tau} d \tau
$$

d'où :

$$
c_{\eta}=\operatorname{tg} \theta c_{\xi}-\tau
$$

ou bien, en substituant (14):

$$
c_{\gamma_{i}}=\operatorname{tg} \theta a \tau /(1+a \tau)-\tau
$$

Une nouvelle integration de $c_{\eta}$ :

$$
\int_{0}^{\eta} d_{\eta}=\operatorname{tg} \theta \quad \int_{0}^{\xi} d \xi \ldots \int_{0}^{\tau} \tau d \tau
$$

nous donne :

$$
\eta=\operatorname{tg} 0 \%-\left(\tau^{2} / 2\right)
$$

et, en substituant (15):

$$
r_{1} / \tau=\operatorname{tg} \theta[1-(1 / a \tau) \ln (1+a \tau)]-(\tau / 2)
$$

Nous passons maintenant à la détermination de la valeur moyenne de la vitesse du grain pendant sa phase de saltation, ce qui est d'ailleurs important pour le problème considéré.

\section{c) Détermination de la vitesse moyenne du grain.}

On a, pour la vitesse moyenne $c_{a m m}$ :

$$
c_{i m}=\left(x_{\max } / t_{\max }\right)=u\left(\xi_{\max } / \tau_{\max }\right)=u c_{\xi m}
$$

Nous trouvons $t_{\max }=\tau_{\text {inax }}(u / g)$ comme seconde racine de l'équation $y=0$ (où $r_{1}=0$ ), c'est-à-dire :

$$
\operatorname{tg} \theta\left[1-\left(1 / a \tau_{\max }\right) \ln \left(1+a \tau_{\max }\right)\right]-\left(\tau_{\max } / 2\right)=0
$$

En posant pour abréger :

$$
z=a \tau_{\max } \text { et } m=1 /(2 a \operatorname{tg} \theta)
$$

nous pouvons ramener cette équation à :

$$
1-m z=(1 / z) \ln (1+z)
$$

c'est-à-dire que la détermination de la durée de saltation se réduit à la solution de l'équation transcendante (19). Exprimées graphiquement, les solutions de cette équation indiquent les abscisses des points d'intersection du faisceau de droites (voir fig. 6) $y=1-m z$ avec la courbe $Y=(1 / z) \ln (1+z)$.

La courbe de la solution $z=\bar{z}(1 / \mathrm{m})$ est représentée sur la figure 7 .

L'allure de la courbe obtenue pour la fonction $z=\bar{z}(1 / m)$ nous permet d'exprimer cette fonction sous la forme :

$$
z=[(1 / m)-2] \lg \alpha_{z}
$$

$\operatorname{tg} \alpha_{z}$ variant elle même en fonction de $z$ (fig. 8).

Etant donné que l'on obtient la longueur de la trajectoire de saltation $\xi_{\max }$ en substituant la 


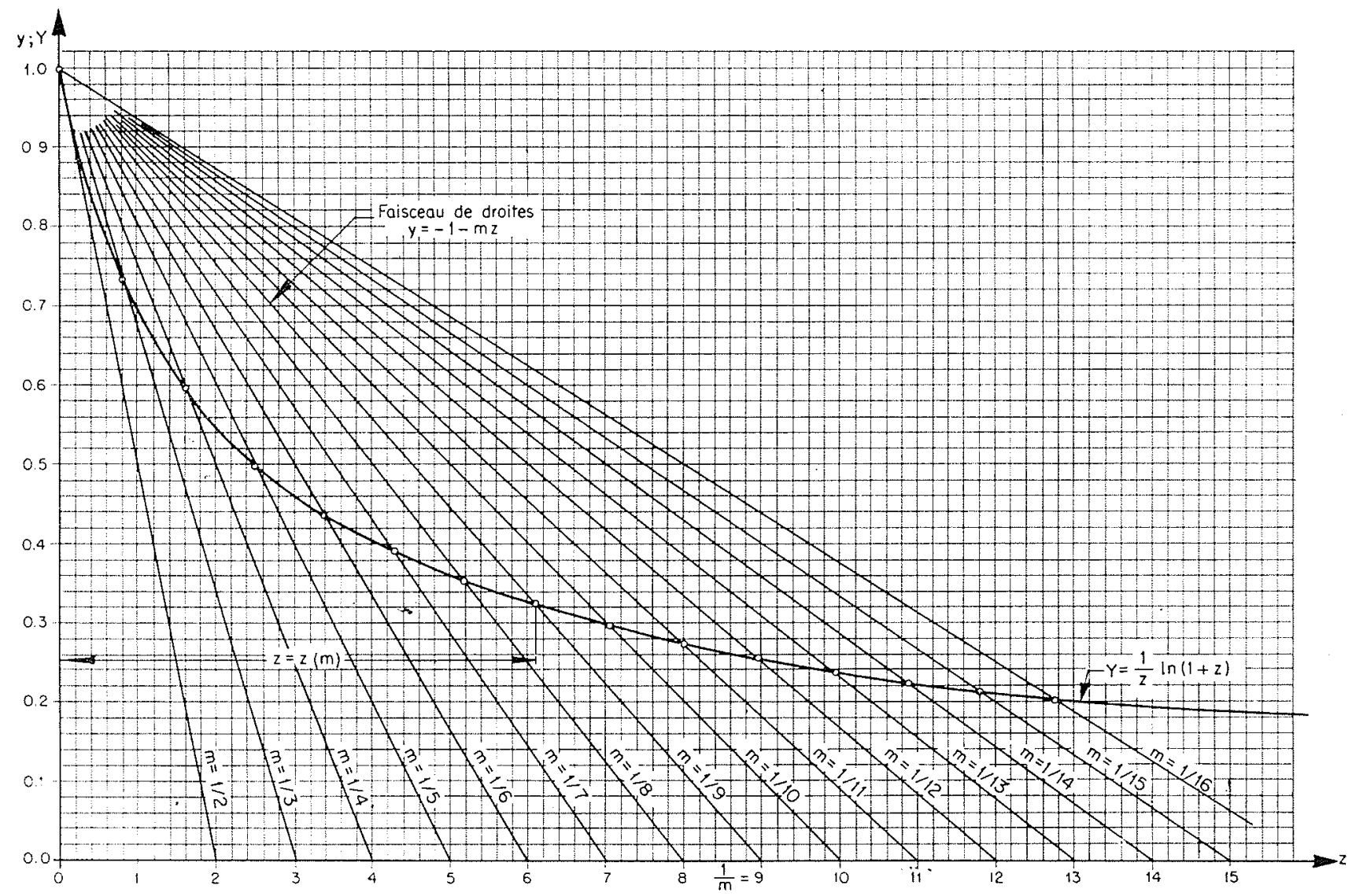

FIG. 6

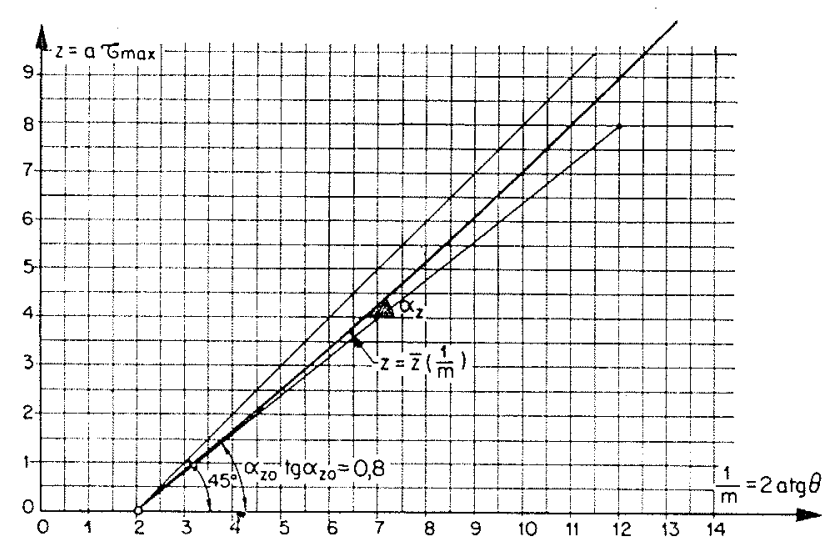

Fid. 7

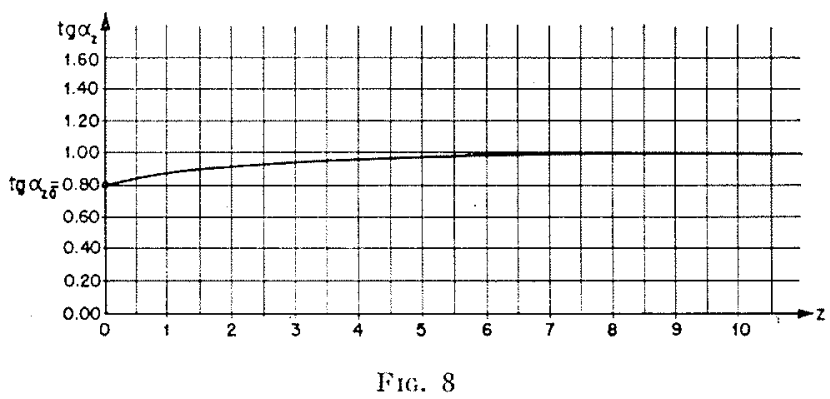

valeur $\tau_{\max }$ dans la formule (15), el cue la valeur $\zeta_{\text {max }} / \tau_{\text {max }}$ est elle-mème égale à $c_{\text {gm }}$, on a :

$$
c_{\xi m}=1-(1 / z) \ln (1+z)
$$

ou bien, en substituant (19):

$$
c_{m}=m z
$$

et, en substituant la valeur $(20)$ de $z$ :

$$
c_{\xi^{m}}=c_{x m} / u=(1-2 m) \operatorname{tg} z_{x}
$$

En négligeant la faible variation de $\lg \%$ au roisinage de 1 , et en posant simplement tg $\alpha_{0}=1$, et finalement en remplaçant $c_{m, n}$ par $c$, on obtient :

$$
c / u=1-2 m
$$

La substitulion de la valeur de $m$ donne finalement:

$$
c / u=1-1 /\left[\sin 0\left(\lambda / \gamma_{f}\right)(\gamma / /) \sigma^{\top}\right]
$$

et comple tenu de la condition rendant la saltation possible :

$$
\mathbf{F}_{y} / G=\sin 0\left(\lambda / x_{f}\right)\left(\gamma / \gamma_{s}\right) \mathscr{F}_{k r}=1
$$


Nous pourons exprimer la formule (21) de la facon suivante :

$$
c / u_{*}=\ddot{a}\left[1-\left(\frac{u_{* w}}{u_{*}}\right)^{2}\right]
$$

ou bien, d'une facon plus générale:

$$
c / \bar{u} \sqrt{g E_{*}}=\sqrt{\mathscr{G}_{*}}\left[1-\left(\mathscr{H}_{* k r} / \mathscr{F}_{*}\right)\right]
$$

\section{d) Débit solide.}

Considérons un élément (grain $d_{i}$ ) du fond de la couche mobile ayant une vitesse moyenne $c_{i}$, et désignons le rapport entre son poids $G_{i}=\alpha_{f} \gamma_{s} d_{i}{ }^{3}$ et l'unité de surface de la projection en plan du fond, par $\Delta \mathcal{G}_{j}$. La valeur absolue de $\Delta \mathcal{G}_{j i}$ n'est évidemment rien d'autre que $G_{i}$; toutefois sa dimension est $\left|\Delta \mathcal{G}_{i}\right|=\mathrm{kg} / \mathrm{m}^{2}$. Si on désigne par $n$ le nombre des grains en mouxement à l'instant considéré sur l'unité de surface $\left(1 \mathrm{~m}^{2}\right)$ du plan de la surface, on peut exprimer le débit solide $q$ par unité de largeur de la couche de fond sous forme de somme des produits $\Delta \mathcal{G}_{i} c_{i}=\Delta q_{i}$ :

$$
q=\sum_{i=1}^{n} \Delta q_{i}=\sum_{i=1}^{n} \Delta \mathcal{G}_{i} c_{i}
$$

ce qui s'explique par des raisons dimensionnelles.

Ou bien, en nous basant sur le théorime des valeurs moyennes :

$$
q=c_{s} \sum_{i=1}^{n} \Delta \mathcal{G}_{i}=c_{s} \mathcal{G}_{\mathcal{J}}
$$

$\mathcal{C}$ étant le poids de toute la masse se trouvant en mouvement à l'instant considéré sur l'unité de surface $d u$ plan du fond, et $c_{s}$ étant la « vitesse de migration $\gg$ de cetle masse-couche.

Choisissons une grandeur linéaire caractéristique quelconque $\delta$ du matériau de fond considéré (telle que $k_{s} ; d_{m} ; d_{\max }$, etc.) et posons $1 \mathrm{~m}^{2}=-\mathrm{B}_{0} \delta^{2}$ pour l'unité de surface, $B_{0}$ représentant un nombre déterminé pour la grandeur choisie $\delta$, nous pouvons alors écrire pour $\Delta \mathcal{G}_{i}$ :

$$
\Delta \mathcal{G}_{i}=\left(\alpha_{f} / B_{0}\right) \gamma_{s} \text { o }\left(d_{i} / \delta\right)^{3}
$$

et pour $\mathcal{G}_{\mathrm{y}}$ :

$$
\mathcal{C}_{z}=\sum_{i=1}^{n} \Delta \mathcal{G}_{h_{i}}=\left(\alpha_{j} / B_{0}\right) \psi_{i} \delta \sum_{i=1}^{n}\left(d_{i} / \delta\right)^{3}
$$

On bien, si nous adoptons les notations :

$$
\mathcal{C}_{\mathfrak{H}, r}=\left(\mu_{i} / \mathrm{B}_{0}\right) \quad \gamma_{s} \text { o et } \Delta \mathrm{N}_{i}=\left(d_{i} / \hat{0}\right)^{3} ; \quad \mathrm{N}=\sum_{i=1}^{\prime \prime} \Delta \mathrm{N}_{i}
$$

nous avons :

$$
\mathcal{G}=\mathcal{G}_{i+1} N
$$

La valeur de $\mathcal{g}_{y}$ est alors entiorement caractérisée par la grandeur sans dimensions $N$ qui croit elle-même à son tour en fonction du nombre $n$ des grains au mètre carré du plan du fond participant au mouvement de masse (12).

Il résulte de la formule (5) que, pour une valeur détermince de l'angle de frottement o et la «géométrie» donnée de la position du grain, l'apparition du décollement d'un stain du fond ne dépend que de la valeur de la Grandeur sans dimensions $\% / 4 \mathscr{G}_{*}\left({ }^{13}\right)$. Ceci veut done dire que, pour une conche de fond ayant une certaine "gémétrie» de surface, le nombre $n$ des grains délachés de cette couche et participant an nouvement de masse, et par suite $N$, ne sont fonction que de la grandeur $\gamma / \gamma_{s} \mathfrak{g}_{\%}$, soil une fonction, croissant uniformément, de $\left(\gamma / \gamma_{s}\right)\left[\mathscr{Y}_{*} \cdots\left(\pi_{*} w_{i r}\right)_{s}\right]$.

Nous choisirons pour cette relation fonctionnelle la forme :

$$
\mathrm{N}=\mathrm{B}_{1}\left[\left(\% / \gamma_{s}\right)\left[\mathscr{H}_{*} \cdots\left(\mathscr{H}_{w}\right)_{s}\right]^{m}\right.
$$

les valeurs de $B_{1}$ et de $m$ ne pouvant ètre déterminées que par voie expérimentale. Etant donné que la configuration des grains a la surface du fond, et par suite la «géomélrie de surface » qui en résulte, dépendent uniquement de la granulométrie du matériau de fond, ceci pour une forme (sphérique) donnée, on comprend que la relation fonctionnelle $N$ (les valeurs $B_{1}$ el $m$ ) doit strictement varier plus ou moins en fonction des allures diverses des courbes sranulométriques.

Nous allons schématiser la relation (24) par une « courbe $N \gg$ (voir fig. 9). Toute entréc d'un «nouveau grain $d_{i}$ queleonque dans le mouvement de masse lorsque la valeur de $\mathscr{F}_{*}$ est $\left(\mathscr{F}_{*} w_{i i}\right)_{i}$ fera augmenter la valeur $\mathrm{N}_{i}$ :

$$
\mathrm{N}_{i}=\mathrm{B}_{1}\left[\left(\gamma / \gamma_{s}\right)\left[\left(\mathscr{F}_{* k r}\right)_{i}-\left(\mathscr{H}_{* / r}\right)_{*}\right]\right]^{m}
$$

d'un incrément $\Delta \mathrm{N}_{i}=\left(d_{i / \delta}\right)^{3}$ et délermineri en outre le point de départ de la courbe de vitesse $c_{i}$ de ce grain. On aura à porter cette courbe sur le schéma tridimensionnel de la figure 9, juste en face de la bande $\Delta N_{i}$ qui lui correspond. La totalité des courbes de c formera ainsi la surface désignée par $\mathcal{S}$ dans la figure 9 . Soit $\iota_{i}\left(\mathscr{H}_{*}\right)$ la valeur de la vitesse moyenne du grain $d_{i}$ pour un certain $\overline{9} *$, le produit élémentaire :

$$
\bar{c}_{i}\left(\mathscr{F H}_{i:}\right) \Delta N_{i}=\Delta S_{i}
$$

qui représente l'élément de surface $J S_{i}$ de la figure 9, est proportionnel à l'élément de débit

(12) Dans le cas particulier des grains de même grosseur $\left(d_{i}=\delta=C^{t e}\right)$, N représenterait simplement le nombre do ces grains $(\mathrm{N}=n)$.

(13) Il est admis que le grain considere se détache dès que la loi quadraticque de la resistance lui devient applicable (roir renvoi 3 ). 


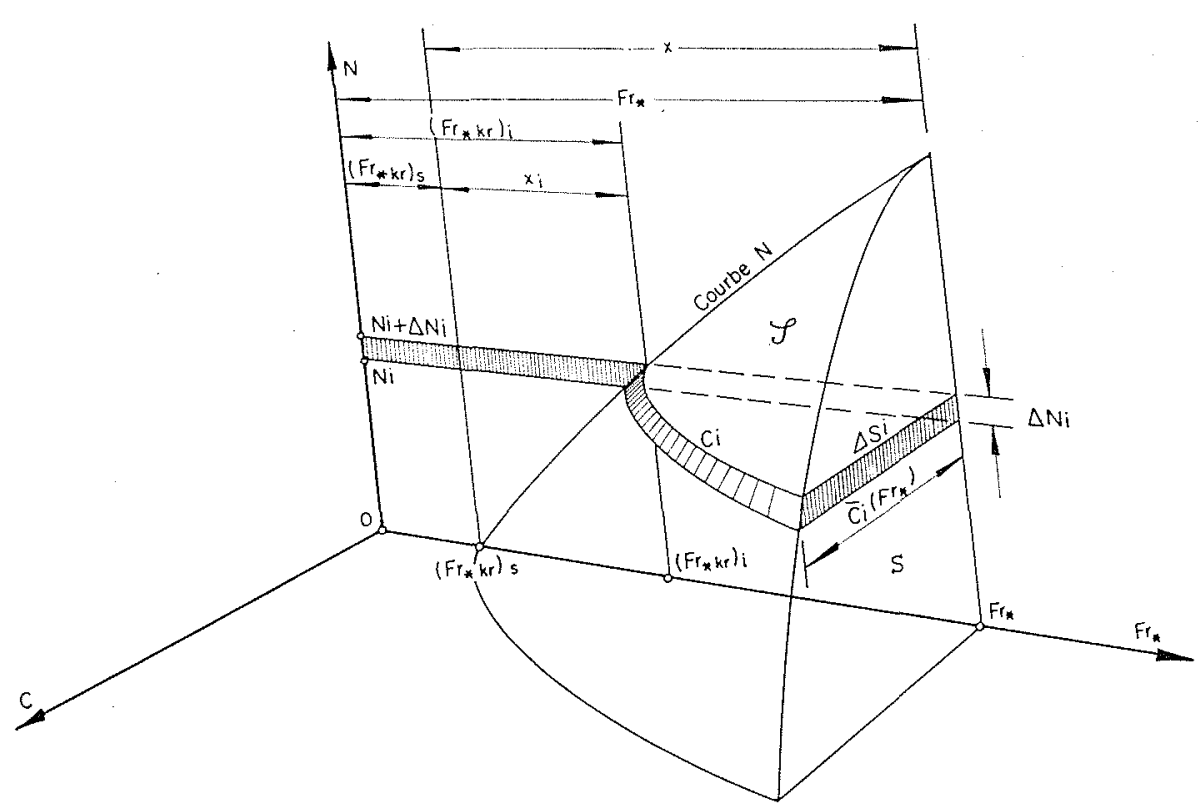

FIG. 9

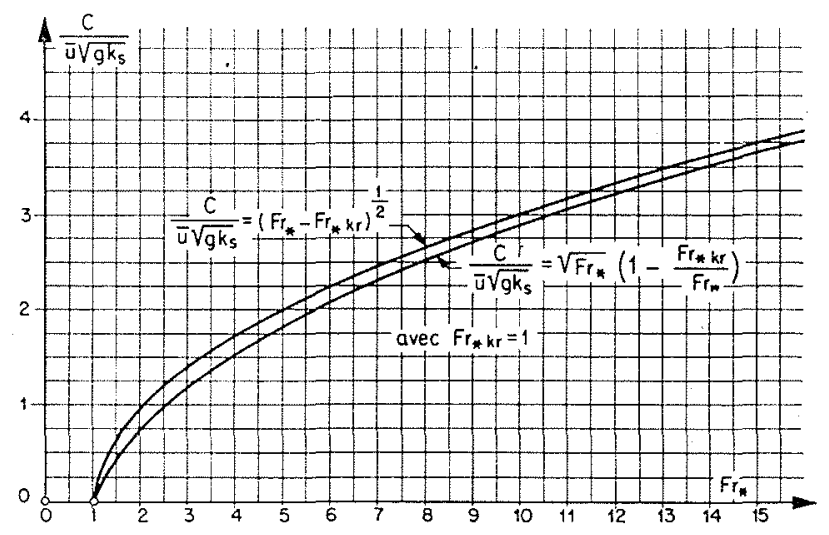

Fig. 10

$\Delta q_{i}$ correspondant au régime d'ćcoulement.g̈. En fail, nous avons, suivant les formules déjà clablies :

$$
\Delta S_{i}=\bar{c}_{i}\left(\mathscr{H}_{k}\right) \Delta \mathrm{N}_{i}=\bar{z}_{i}\left(\mathscr{H}_{*}\right)\left(\Delta \mathcal{g}_{i} / \mathcal{G}_{0}\right)=\left(1 / \mathcal{G}_{0}\right) \Delta q
$$

el la somme de ces élements devient:

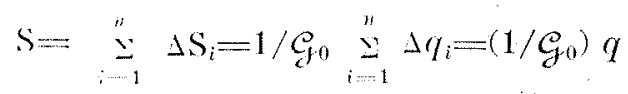

On voit que les aires des sections $\mathrm{S}$ correspondant à des $\mathscr{F}_{*}$ déterminés sont proportionnelles aux débits solides du fond considéré correspondant à la même valeur de $\mathscr{F}_{*}$.

Comple tenu du fait que la difírence entre les deux courbes de la figure 10 est entièrement négligeable dans le cas considéré, nous pouvons remplacer l'expression (21 2 ) par une autre qui convient pratiquement aussi bien à nos fins et qui est beaucoup plus avantageuse du point de vue des operations mathématiques que nous avons à effectuer :

$$
c / u \sqrt{g l_{s}}=\left(\mathscr{H}_{*}-\mathscr{J H}_{* k r}\right)^{1 / 2}
$$

avec $\mathscr{H}_{: k i r}=\left(\mathscr{H}_{*, r}\right)_{i}$. La somme ci-dessus s'écrira donc :

$$
\left(1 / \mathcal{G}_{0}\right) q=\left(\tilde{i} \bar{V} \sqrt{g k_{s}}\right) \sum_{i=1}^{n}\left[\mathscr{H}_{*}-\left(\mathscr{H}_{* k r}\right)_{i}\right]^{1 / 2} \Delta N_{i}
$$

En passant à la valeur limite, nous avons, pour $\Delta N_{i}$ :

$$
\begin{array}{r}
\Delta \mathrm{N}_{i}=d \mathrm{~N}_{i}=m \mathrm{~B}_{1}\left(\gamma / \gamma_{s}\right)^{m}\left[\left(\mathscr{H}_{* k_{r} r}\right)_{i}-\left(\mathscr{H}_{* k}\right)_{s}\right]^{m-1} \\
d\left[\left(\mathscr{H}_{* k}\right)_{i}-\left(\mathscr{H}_{*} * w\right)_{s}\right]
\end{array}
$$

et pour notre somme :

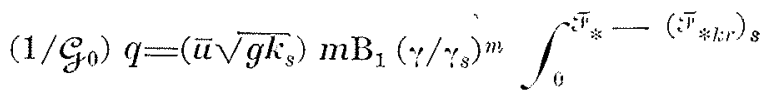

$$
\begin{aligned}
& {\left[\mathscr{H}_{*}-\left(\mathscr{H}_{* l r}\right)_{i}\right]^{1 / 2}\left[\left(\mathscr{H}_{* k r}\right)_{i}-\left(\mathscr{H}_{* k r}\right)_{s}\right]^{m-1}} \\
& d\left[\left(\mathscr{H}_{* k_{i r}}\right)_{i}-\left(\mathscr{H}_{* k i r}\right)_{s}\right]
\end{aligned}
$$

Substituant la valeur $\mathcal{G}_{0}$ et les expressions :

$$
\left(\mathscr{H}_{* k r}\right)_{i}-\left(\mathscr{H}_{* k i}\right)_{s}=x_{i} ; \mathscr{H}_{*}-\left(\mathscr{H}_{* k, r}\right)_{s}=x ; \hat{o}=\varkappa_{0} k_{s}
$$

nous obtenons :

$q=\mathrm{B}_{2} m\left(y^{m / y_{s}}{ }^{m-1}\right) g^{1 / 2} k_{s}+2 \int_{0}^{x}\left(x-x_{i}\right)^{1 / 2} x_{i}^{m-1} d x_{i}$ 
avec :

$$
\mathrm{B}_{2}=\left(\mathrm{B}_{1} / \mathrm{B}_{0}\right) \alpha_{\mathrm{f}} \alpha_{0} \bar{u}
$$

On voit, d'apres l'expression (25), que l'intigration donne :

$$
\ldots \ldots x^{m+(1 / 2)}
$$

comme « terme maximum », ce qui signifie done

$$
\mathscr{W}_{*:}^{m+(1 / 2)}
$$

Compte tenu de la fonction quadratique :

$$
\mathscr{H}_{*}=n_{*}{ }^{2} / g k_{s}
$$

nous pouvons conclure que la valeur de $q$ exprimée par $l_{*}$ contiendra le «terme maximum》:

$$
\left.\ldots u_{*}\right)^{m+(1 / 2)}=\ldots u_{*}^{2 m+1}
$$

On trouve, par comparaison avec les résullats expérimentaux [voir la formule empirique dans le renvoi $\left.\left({ }^{* *}\right)\right]$, que le nombre $m$ doit évidemment avoir une valeur voisine de 1 . Si on admet simplement que $m=1$ (c'est-à-dire la croissance linéaire de la masse mobile en fonction de la force d'entrainement), on a pour (25) :

$$
q=B_{2} q^{1 / 2} k_{s}^{3 / 2} \int_{0}^{u}\left(x-x_{i}\right)^{1 / 2} d x_{i}
$$

D'où nous obtenons, après intégration :

$$
q=\mathrm{B} * g^{1 / 2} k_{s}^{3 / 2} x^{3 / 2} \text { avec } \mathrm{B}=2 / 3 \mathrm{~B} .
$$

Substituant la valeur de $x$, nous trourons l'expression requise pour le débit solide :

$$
\begin{aligned}
& q=-\mathrm{B} \gamma q^{1 / 2} k_{s}: 3 / 2\left[\mathscr{H}_{*}-\left(\mathscr{H}_{* k r}\right)_{s}\right]^{3 / 2}=\mathrm{B}(\gamma / g) \\
& {\left[u_{*}^{2} \cdots\left(u_{* N N_{*}}\right)^{2}\right]^{* / 2}}
\end{aligned}
$$

ou bien :

$$
q=\mathrm{B}\left(g^{\prime} / \gamma^{1 / 2}\left(\tau_{0}-\tau_{(1 / i}\right)^{n / 2}\right.
$$

Si on exprime $\tau_{0}$ et $\tau_{0} \ldots$ par leurs valeurs iespectives $(7)$ et $(8)$, on a :

$$
q=\mathrm{B}(g / \gamma)^{1 / 2}\left[\gamma^{\prime} h i-\mathrm{A}{ }_{s} d_{m}\right]^{*}
$$

ce cui concorde, aux valeurs numeriques des coefficients pris, avec la formule de Meyer-Peler pour le débit bidimensiomel :

$$
\eta=8(g / \gamma)^{1 / 2}\left(\gamma / v i-0,047 \gamma_{s} d_{m}\right)^{n / 2}
$$

En conclusion, signalons que, en toule rigueur, les valeurs des « constantes » dans la formule suisse, c'est-à-dire : $B=8$ et $A=0,047$, varient plus ou moins en fonction de l'allure des courbes granulométriques (ce qui se comprend d'après la signification donnce à ces grandeurs par la présente étude), de sorte qu'elles différeront des valeurs ci-dessus, qui ont été déterminées exclusivement pour des matériaux de fond ayant des courbes granulométriques conformes au type « $a »$ de la figure $4\left(^{14}\right)$.

(14) S. Meven-Peter, Mürefn : Einc Formel zux Berechnung des Geschicbetriebes, Schweiz, Banzeitung, 6ī, Nr. 3, 1949 .

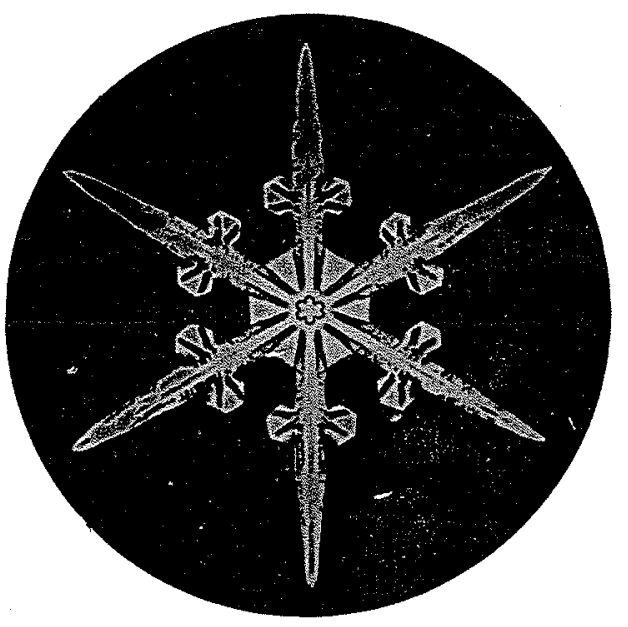

